\title{
Reconfigurable Parallel Turbo Decoder Design for Multiple High-Mobility 4G Systems
}

\author{
Cheng-Hung Lin • Chun-Yu Chen • En-Jui Chang • \\ An-Yeu Wu
}

Received: 28 June 2012 / Accepted: 4 February 2013 / Published online: 28 February 2013

(C) Springer Science+Business Media New York 2013

\begin{abstract}
For high-mobility 4G applications of LTE-A and WiMAX-2 systems, this paper presents a dual-standard turbo decoder design with the following three techniques. 1) Circular parallel decoding reduces decoding latency and improves throughput rate. 2) Collision-free vectorizable dual-standard parallel interleaver enhances hardware utilization of the interleaving address generator. 3) One-bank extrinsic buffer design with bit-level extrinsic information exchange reduces size of the extrinsic buffer compared with the two-bank extrinsic buffer design. Furthermore, a multistandard turbo decoder chip is fabricated in a core area of $3.38 \mathrm{~mm}^{2}$ by $90 \mathrm{~nm}$ CMOS process. This chip is maximally measured at $152 \mathrm{MHz}$ with $186.1 \mathrm{Mbps}$ for LTE-A standard and 179.3 Mbps for WiMAX-2 standard.
\end{abstract}

Keywords Turbo codes $\cdot$ WiMAX $\cdot$ LTE $\cdot$ Multi-standard

This work was supported in part by National Science Council, R.O.C., under grants NSC 99-2218-E-155-011 and NSC 97-2220-E-002-012. The material in this paper was presented in part at the IEEE ISIC 2011, Singapore.

\section{C.-H. Lin $(\square)$}

Department of Electrical Engineering, Yuan Ze University, Jungli 32003, Taiwan, Republic of China

e-mail: chlin@saturn.yzu.edu.tw

E.-J. Chang - A.-Y. Wu

Graduate Institute of Electronics Engineering and Department of Electrical Engineering, National Taiwan University, Taipei 10617, Taiwan, Republic of China

A.-Y. Wu

e-mail: andywu@cc.ee.ntu.edu.tw

C.-Y. Chen

Silicon Motion Technology Corp., Sindian 23141, Taiwan, Republic of China

\section{Introduction}

Convolutional turbo code (CTC) has been a regular forward error correction (FEC) scheme for reliable wireless communications with a rapid growth of multimedia services. Single-binary CTC (SB-CTC) proposed in 1993 achieves high data rates and coding gains close to the Shannon limit [1]. SB-CTC has been adopted in 3rd Generation Partnership Project (3GPP) family standards [2] as an FEC scheme because of its good correction performance. Non-binary CTC (NB-CTC) [3] introduced in 1999 has superior coding performance than the SB-CTC. In recent years, double-binary CTC (DB-CTC) was adopted in Worldwide Interoperability for Microwave Access (WiMAX) family standards [4] as a FEC scheme. Table 1 lists the detailed specifications and CTC schemes of the prevalent wireless standards for wide area networks (WANs).

Recently, there are a large growing emergence and demand for an inexpensive and ubiquitous broadband wireless network. Thus, Long Term Evolution (LTE) and WiMAX standards become prevalent for the broadband wireless network. Meanwhile, fourth generation (4G) cellular wireless communication, the term referred to International Mobile Telecommunications-Advanced (IMT-Advanced) [5], is emerging in high-end broadband wireless devices. Nowadays the $4 \mathrm{G}$ compliant versions of LTE and WiMAX are LTE Advanced (LTE-A) and WirelessMAN-Advanced (WiMAX-2), respectively. To achieve a smooth migration for different applications, a CTC decoder that works across the dual IMT-Advanced compliant standards is necessary. Hence, the goal of this work is to design a CTC decoder that can be used in high-mobility $4 \mathrm{G}$ communications. The features of the CTC decoder design are 
Table 1 Specifications of the prevalent wireless wide area networks.

\begin{tabular}{llllll}
\hline Standard & \multicolumn{2}{l}{ 3GPP family } & & \multicolumn{2}{l}{ WiMAX family } \\
\cline { 2 - 3 } \cline { 5 - 6 } & LTE & LTE-A & & WiMAX & WiMAX-2 \\
\hline Specification & Rel.8 & Rel.10 & & $802.16 \mathrm{~d}$ & $802.16 \mathrm{~m}$ \\
Max. data rate & $100 \mathrm{Mbps}$ & $100 \mathrm{Mbps}$ (High Mobility) & & $75 \mathrm{Mbps}$ & $100 \mathrm{Mbps}$ (High Mobility) \\
Bit error rate & $10^{-5}$ & & & $10^{-5}$ & \\
CTC scheme & Single-binary (SB) CTC & & Double-binary (DB) CTC \\
Inf. Bits/Block & $64 \sim 6144$ & $48 \sim 4800$ & \\
\hline
\end{tabular}

- to achieve the high-throughput requirement targeting at the specifications of LTE-A and WiMAX-2 standards shown in Table 1,

- to achieve parallel decoding for SB-CTC and DB-CTC schemes based on LTE-A and WiMAX-2 standards,

- to design a parallel and reconfigurable interleaver based on the computational similarity of LTE-A and WiMAX2 standards, and

- to achieve area-efficient and memory-efficient CTC decoder design for the LTE-A and WiMAX-2 standards.

In this paper, circular parallel maximum a-posteriori probability algorithm (MAP) decoding is introduced to reduce decoding latency and hardware cost. The circular parallel MAP decoding results in numbers of circular MAP decoding according to its parallelism with a small coding gain loss. Meanwhile, the collision-free vectorizable dual-standard parallel interleaver based on the almost regular permutation (ARP) structure and the quadratic polynomial permutation (QPP) structure is proposed to enhance hardware utilization of the interleaver. Then, the available parallelism of the collision-free CTC decoding is achieved for the LTE-A and WiMAX-2 standards. In order to increase the hardware usage of the CTC decoder, two area-efficient extrinsic buffer designs are described for the dual-standard CTC decoder. Using UMC $90 \mathrm{~nm}$ CMOS technology, the proposed CTC decoder chip for WiMAX-2 and LTE-A systems has been fabricated in a core size of $3.38 \mathrm{~mm}^{2}$. The throughput rate of $186.1 \mathrm{Mbps}$ can be maximally measured at $152 \mathrm{MHz}$ with power consumption of $148.1 \mathrm{~mW}$.

This paper is organized as follows. In Section 2, the fundamentals of turbo codes for WiMAX-based standard and LTEbased standard are revisited. Section 3 describes the architecture design of the proposed CTC decoder for the dual standards. Section 4 presents the experimental results and comparison of the proposed CTC decoder for the LTE-A/WiMAX-2 standards. Finally, Section 5 concludes this paper.

\section{Reviews of Turbo Codes}

Background reviews of the CTCs of WiMAX-based standard and LTE-based standard are demonstrated in this section.

\subsection{Turbo Codec}

The encoder and decoder of CTC are shown in Fig. 1. The encoder consists of two identical recursive systematic convolutional (RSC) encoders, which are connected in parallel by an interleaver. The RSC encoder produces systematic symbol $\left(u_{s}\right)$ and parity symbol $\left(u_{p}\right)$ to the channel. The information stream is reordered by the interleaver and then enters the second RSC encoder. The RSC encoders of SBCTC encodes 1 bit per time while the RSC encoders of DBCTC encodes 2 bits per time. The RSC encoders of LTE (SB-CTC) and WiMAX (DB-CTC) standards are shown in Fig. 2. For the trellis termination, the RSC encoder in LTE uses a few redundant bits to terminate the trellis path. However, the RSC encoder in WiMAX adopts two-phase circular encoding [3] where the final trellis state can be the same to initial trellis state.

The decoder is composed of two soft-input soft-output (SISO) decoders that are serially concatenated by the interleaver and de-interleaver. Each SISO decoder uses the received systematic symbol and corresponding parity symbol to computes extrinsic information that is then iteratively fed to the other SISO decoder as the a priori information. The hard decision of decoded symbols is made after several iterations between these two SISO decoders.

\subsection{CTC Interleaver}

The interleaver is one of dominant modules of CTCs and the correction performance of CTCs depends on the structures and length of interleaver. The interleaver is used to permute the order of symbols. This can be done by using an interleaving address generator to access the symbols stored in a buffer. When the $c_{i}$ denotes normal-order symbol, the interleaved- order symbol $c_{\pi(i)}^{\prime}$ can be represented as

$c_{i}^{\prime}=c_{\pi(i)}$,

where $i=0,1, \ldots,(N-1)$ and $N$ is the CTC block size. $c_{\pi(i)}$ is the normal-order symbol $c_{i}$ stored at interleaving pattern $\pi(i)$ and $\pi(i)$ is generated by interleaving address generator. The algorithms that generate $\pi(i)$ are defined in each standard and described as follows. 
a

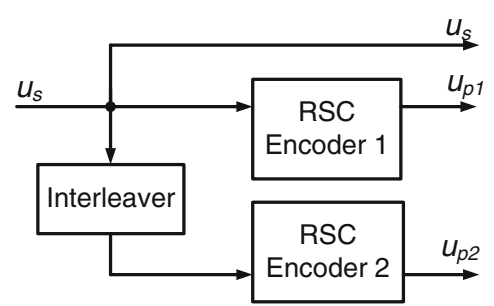

b

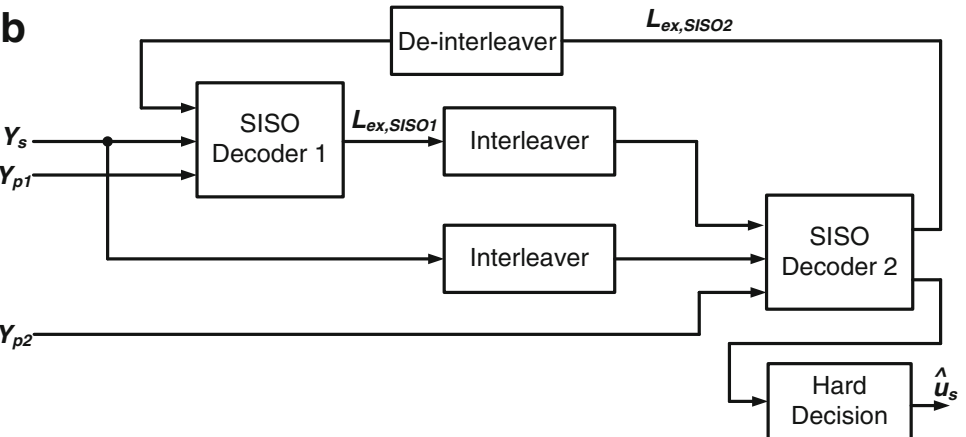

Figure 1 Block diagram of a CTC encoder and b CTC decoder.

The WiMAX interleaver adopts the ARP structure. The interleaving address $\pi_{W}(j)$ is generated by switching $(j)_{\bmod 4}$ :

$$
\begin{aligned}
& \text { case } 0: \pi_{\mathrm{W}}(j)=\left(P_{0} \times j+1\right)_{\bmod N}, \\
& \text { case } 1: \pi_{\mathrm{W}}(j)=\left(P_{0} \times j+1+P_{1}\right)_{\bmod N}, \\
& \text { case } 2: \pi_{\mathrm{W}}(j)=\left(P_{0} \times j+1+N / 2+1+P_{2}\right)_{\bmod N,}, \\
& \text { case } 3: \pi_{\mathrm{W}}(j)=\left(P_{0} \times j+1+N / 2+1+P_{3}\right)_{\bmod N},
\end{aligned}
$$

where $j$ is an increasing value from 1 to $N$. The parameters $P_{0}, P_{1}, P_{2}$, and $P_{3}$ defined in WiMAX depend on $N$. The function of $(x)_{\bmod y}$ gets the remainder on division of $x$ by $y$. The parameters $P_{0}, P_{1}, P_{2}$, and $P_{3}$ can be stored into a lookup table (LUT). The individual architecture of WiMAX CTC interleaver can be referred to in [6].

The LTE interleaver adopts the QPP structure. The interleaving address $\pi_{L}(j)$ is generated by

$\pi_{L}(j)=\left(f_{1} \times j+f_{2} \times j^{2}\right)_{\bmod N}$,

where $j$ is an increasing value from 1 to $N$. The parameters $f_{1}$ and $f_{2}$ defined in LTE also depend on $N$. The parameters $f_{1}$ and $f_{2}$ can be stored into a LUT as well. The individual architecture of LTE CTC interleaver can be referred to in [7].

\subsection{MAP Decoding for SB/DB CTC}

Maximum a-posteriori probability algorithm (MAP) [8] and its derivatives [9-11] are widely employed in the CTC decoding. Furthermore, the enhanced Max-log-MAP (EML-MAP) proposed in [11] is achieved with a little coding gain degradation for an ease of hardware implementation of the MAP. In the following sections, we use the name MAP for a short abbreviation of the EML-MAP. Given the received block sequence $Y$, the MAP gives each decoded bit $u_{k}$ a probability that the bit is 1 or 0 . This is equivalent to find a-posteriori loglikelihood-ratio (LLR),

$\Lambda_{\text {apo }}\left(u_{k}\right)=\ln \left(\frac{\operatorname{Pr}\left(u_{k}=1 \mid Y\right)}{\operatorname{Pr}\left(u_{k}=0 \mid Y\right)}\right)$.

$\Lambda_{\text {apo }}$ can be decomposed as follows

$\Lambda_{\text {apo }}\left(u_{k}\right)=\ln \left(\frac{\sum_{\left(s^{\prime}, s\right) \Rightarrow u_{k}=1}\left(\alpha_{k}\left(s^{\prime}\right) \cdot \gamma_{k}\left(s^{\prime}, s\right) \cdot \beta_{k+1}(s)\right)}{\sum_{\left(s^{\prime}, s\right) \Rightarrow u_{k}=0}\left(\alpha_{k}\left(s^{\prime}\right) \cdot \gamma_{k}\left(s^{\prime}, s\right) \cdot \beta_{k+1}(s)\right)}\right)$,

$\alpha_{k+1}(s)=\sum_{\text {all } s^{\prime}}\left(\alpha_{k}\left(s^{\prime}\right) \cdot \gamma_{k}\left(s^{\prime}, s\right)\right.$,

$\beta_{k}\left(s^{\prime}\right)=\sum_{\text {all } s^{\prime}}\left(\beta_{k+1}(s) \cdot \gamma_{k}\left(s^{\prime}, s\right)\right.$,

$\gamma_{k}\left(s^{\prime}, s\right)=\operatorname{Pr}\left(y_{k} \mid x_{k}\right) \cdot \operatorname{Pr}\left(u_{k}\right)$,

where $\alpha$ is the forward state metrics; $\beta$ is the backward state metrics; $\gamma$ is the branch metrics; $k$ is the decoding time index; $s$,
Figure 2 Block diagram of the a SB RSC encoder in LTE and b DB RSC encoder in WiMAX. a

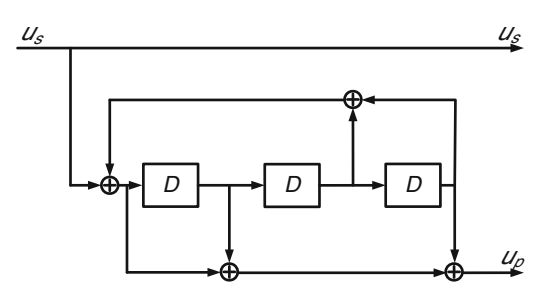

b

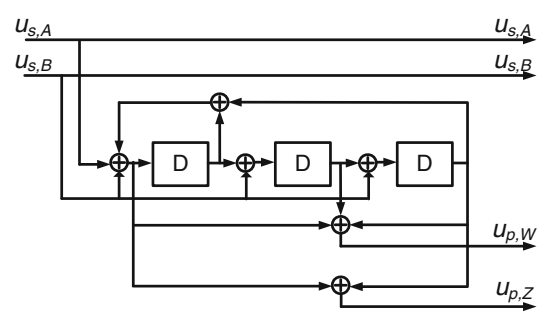




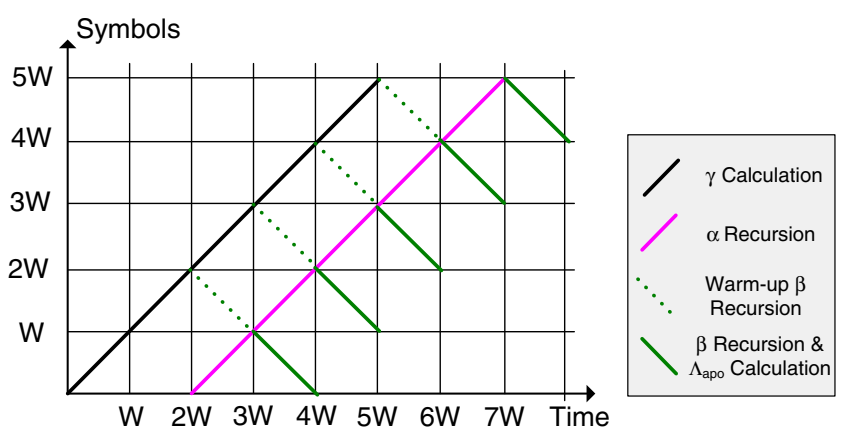

Figure 3 Warm-up sliding-window (SW) MAP decoding.

$s^{\prime}$ denotes the state indices; $\operatorname{Pr}\left(\underline{y}_{k} \mid \underline{x}_{k}\right)$ is the conditional received symbol probability; and $\operatorname{Pr}\left(\underline{u}_{k}\right)$ is the a priori probability of decoded bit $u_{k}$ attained from the other SISO decoder.

During the turbo decoding, extrinsic information is iteratively fed back to the other SISO decoder as a priori LLR. The extrinsic information $\Lambda_{e x}$ is formulated as

$\Lambda_{\text {ex }}\left(u_{k}\right)=\delta\left(\Lambda_{\text {apo }}\left(u_{k}\right)-\Lambda_{\text {apr }}\left(u_{k}\right)-\Lambda_{\text {in }}\left(u_{k}\right)\right)$,

where $\Lambda_{\text {in }}$ and $\Lambda_{\text {apr }}$ are the intrinsic information and a priori LLR of this SISO decoding, respectively. The extrinsic information in the EML-MAP multiplies a scaling factor $(0<\delta<1)$. For either the SB-CTC or DB-CTC decoding, the MAP is composed of branch metrics $(\gamma)$, forward recursion state metrics $(\alpha)$, backward recursion state metrics $(\beta)$, a priori $\operatorname{LLR}\left(\Lambda_{\text {apr }}\right)$, a posteriori $\operatorname{LLR}\left(\Lambda_{\text {apo }}\right)$, and extrinsic information $\left(\Lambda_{e x}\right)$. For the dual-standard MAP decoding, the radix-4 SB MAP decoding and radix-4 DB MAP decoding are employed because of their similarity. The details of radix-4 SB MAP decoding and radix-4 DB MAP decoding can be referred to in [12].

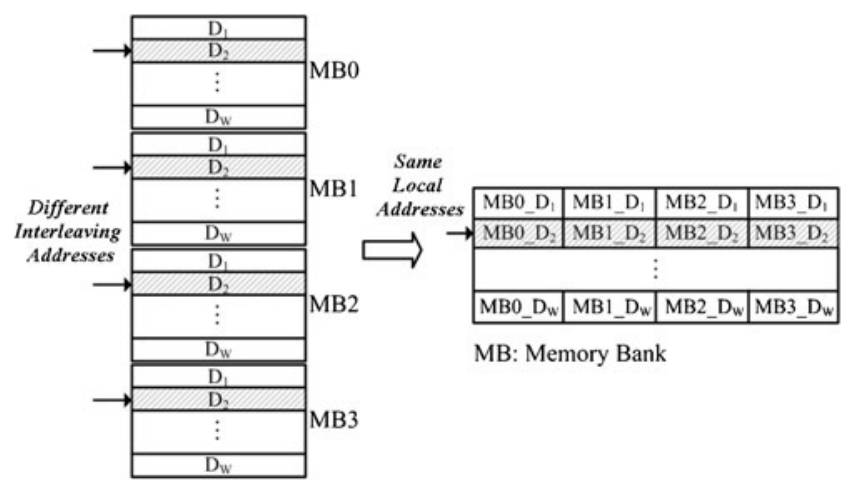

Figure 5 Vectorizable interleaving address for memory banks.

\subsection{Window-Based MAP Decoding}

The windowing technique proposed in [13] is used to facilitate the memory cost of CTC decoders. The sliding window (SW) decoding [14] deals with any CTC block size but has an intrinsic low throughput rate. Some VLSI architectures of the sliding window (SW) MAP decoding can be referred to in [15-18]. Figure 3 shows the timing chart of the warm-up SW MAP decoding, and the vertical and horizontal axes denote the decoding symbol and decoding time, respectively. The timing chart of MAP decoding is mainly composed of the branch metric acquisition, forward state metrics recursion, backward state metrics recursion, and a posteriori LLR acquisition. In order to achieve the reliable window rim, the warm-up recursion of a basic windows size $W$ is performed. In general, $W=4 v \sim 6 v$, where $v$ is the constraint length of RSC encoder. The decoding latency of the warm-up SW MAP decoding is 3 $W$. The SW MAP decoding deals with any CTC frame size but it has an intrinsic low throughput. The hybrid-window (HW)

Figure 4 Block diagram of the proposed CTC decoder for LTE-A/WiMAX-2 Standards.

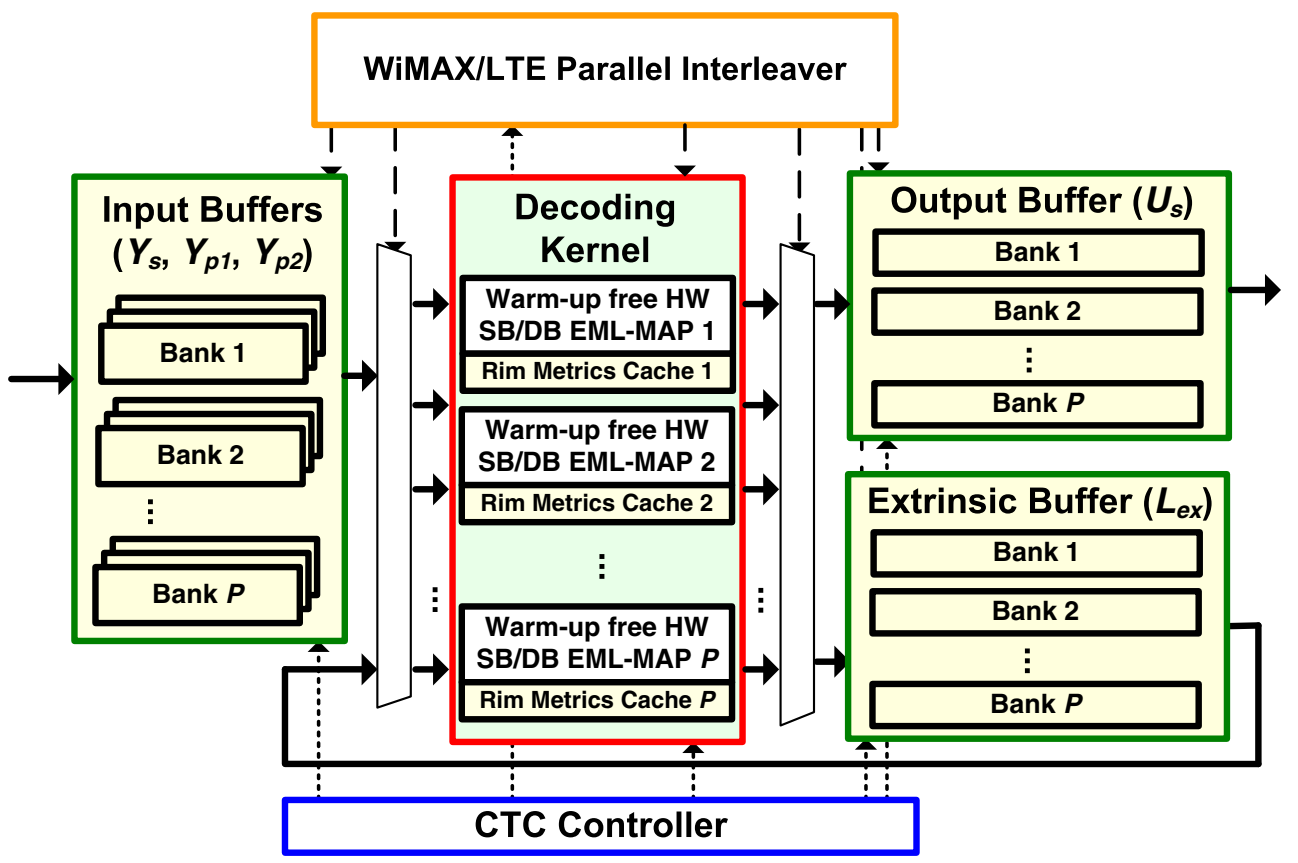


MAP decoding described in [12] applies parallel decoding with parallelism $P$ to decode one received block. Because of the warm-up processes of forward states metrics, the decoding latency is prolonged to $4 W$. Nevertheless, the HW MAP decoding can shorten the decoding cycles to $N / P+4 W$ by working with several sub-blocks simultaneously.

\section{Proposed CTC Decoder for LTE-A/WiMAX-2 Standards}

In order to support the decoding of both WiMAX-based and LTE-based CTC schemes, our realization of the proposed dual-standard CTC decoder is based on the architecture shown in Fig. 4. The input buffers store the soft received data including the systematic information, non-interleaved parity information, and interleaved parity information. Meanwhile, the internal buffer stores the extrinsic information and the output buffer stores the decoded hard bits. Because at most $P$ warm-up free HW (WFHW) MAP processors work concurrently, each buffer is divided into $P$ banks to be accessed simultaneously. The proposed LTE/WiMAX parallel interleaver generates collision-free vectorizable addresses for the input buffer and extrinsic buffer. Therefore, the normal-order or interleaved-order data can be correctly processed between the WFHW MAP processors and the buffer subbanks. When the targeted iteration number is reached or the hard bits of two half iteration are the same,

Table 2 Available parallelism for LTE/WiMAX systems.

\begin{tabular}{|c|c|c|}
\hline \multirow[t]{2}{*}{ Inf. Bits } & \multicolumn{2}{|l|}{ Available parallelism $P$} \\
\hline & WiMAX & LTE \\
\hline 48 & 1 & 12 \\
\hline 72 & 1 & 123 \\
\hline 96 & 12 & 1234 \\
\hline 144 & 123 & 12346 \\
\hline 192 & 1234 & 123468 \\
\hline 216 & 13 & 1234689 \\
\hline 240 & 12345 & 123456810 \\
\hline 288 & 12346 & 123468912 \\
\hline 360 & 123456 & 12345689101215 \\
\hline 384 & 123468 & 1234681216 \\
\hline 432 & 1234689 & 1234689121618 \\
\hline 480 & 123456810 & 12345681012151620 \\
\hline 960 & 12345681012 & 12345681012151620 \\
\hline 1920 & 12345681012 & 12345681012151620 \\
\hline 2880 & 123456891012 & 12345689101215161820 \\
\hline 3840 & 12345681012 & 12345681012151620 \\
\hline 4800 & 12345681012 & 12345681012151620 \\
\hline 6144 & - & 12345681216 \\
\hline
\end{tabular}

the CTC decoder finishes the decoding procedure and outputs the hard bits from the output buffer. The design techniques are presented in detail as follows.

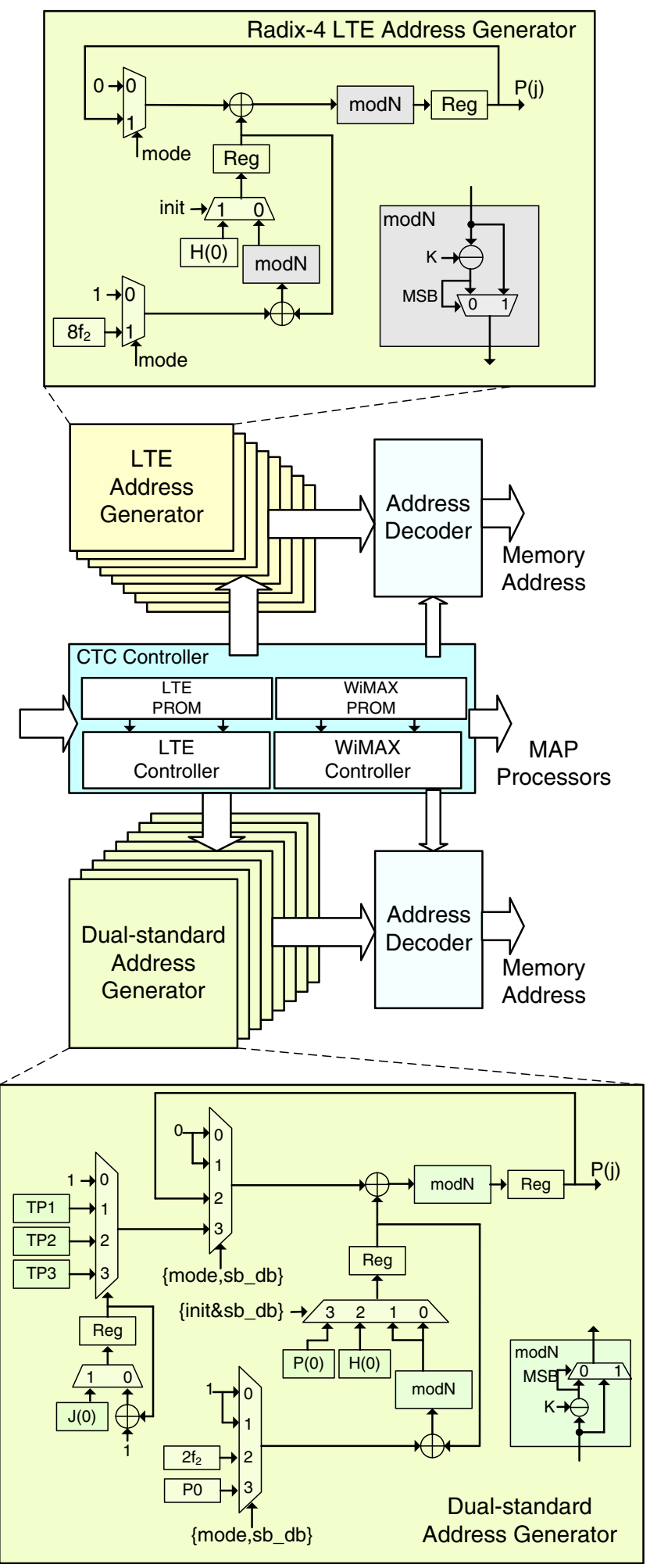

Figure 6 Block diagram of the LTE/WiMAX parallel interleaver and CTC controller. 
Table 3 Gate-count analysis of the proposed collision-free vectorizable LTE/WiMAX parallel interleaver and CTC controller.

\begin{tabular}{lc}
\hline Modules & \multicolumn{1}{l}{ Gate counts } \\
\hline CTC controller & $8.0 \mathrm{~K}(26.3 \%)$ \\
Dual-standard address generators & $6.9 \mathrm{~K}(22.7 \%)$ \\
LTE address generators & $5.7 \mathrm{~K}(18.8 \%)$ \\
Address decoders & $9.8 \mathrm{~K}(32.2 \%)$ \\
Overall & $30.4 \mathrm{~K}(100.0 \%)$ \\
\hline
\end{tabular}

\subsection{Collision-Free Vectorizable WiMAX/LTE Parallel Interleaver}

The CTC interleaving is used to permute symbols by an interleaving address generator that accesses symbols from the buffers. For parallel decoding, $P$ MAP processors may read and write a same memory bank simultaneously. Since the port of a memory is finite, the simultaneous memory access is prohibited. Without a cautious analysis of the LTEbased and WiMAX-based CTC interleaving, the memory collision occurs frequently and makes the parallel decoding unrealizable [19]. Finding a proper parallelism $P$ has been discussed in [20]. The parallel interleaver is collision-free when it satisfies

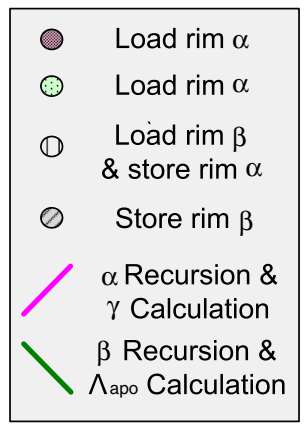

a

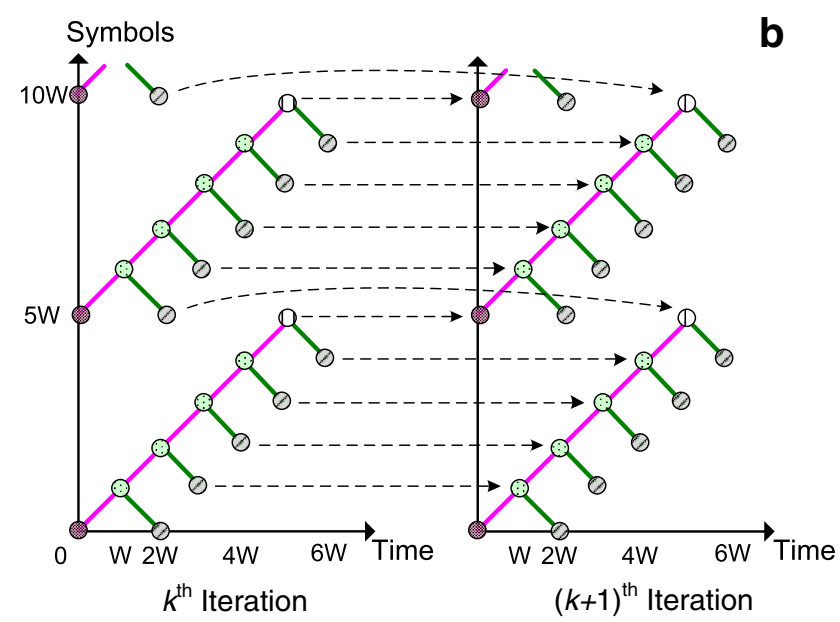

Figure 7 Warm-up free hybrid-window (WFHW) MAP decoding: a basic window and $\mathbf{b}$ overall timing chart.

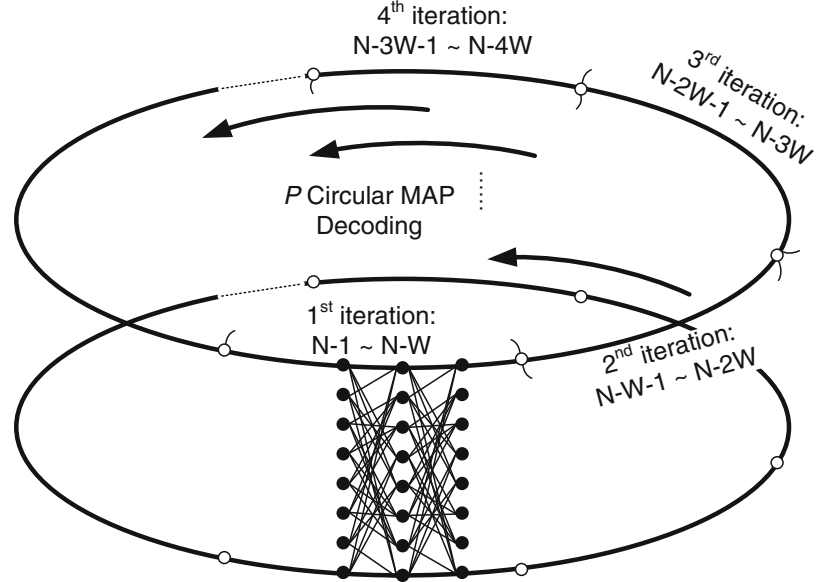

Figure 8 Equivalent trellis propagation of backward recursion of the circular parallel MAP decoding as the iteration is increased.

$\left\lfloor\frac{\pi(t+j W)}{W}\right\rfloor \neq\left\lfloor\frac{\pi(t+k W)}{W}\right\rfloor$,

where $0 \leqq t<W$, and $0 \leqq j, k<P$. The terms on both sides in (7) are indices of the memory banks that are accessed by the $j^{\text {th }}$ and $k^{\text {th }}$ MAP processors at the $t^{\text {th }}$ time instant. This inequality need to be true for any time instant $t$ for no memory collision.

For an interleaver design, the complexity of the interleaving address generation is also critical. Each memory bank requires an address decoder to transform the global interleaving address to the local address for each memory bank. As the parallelism $P$ increases, the duplication of address decoder leads to hardware inefficiency. A better

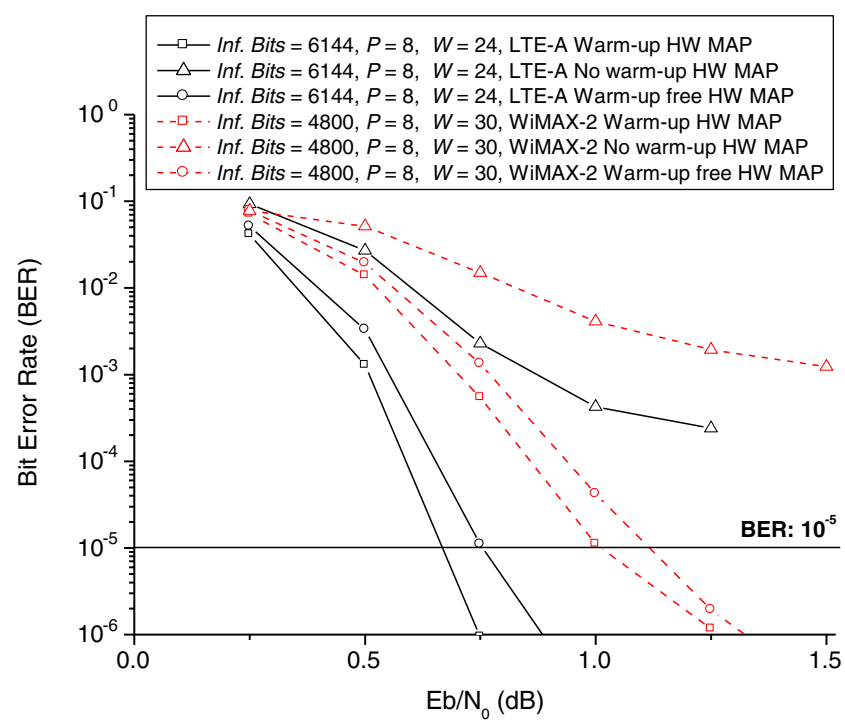

Figure 9 BER performance of the WiMAX-2 and LTE-A CTC decoding by using the distinct hybrid-window (HW) MAP decoding at fixed 6 iterations. "No warm-up" represents that the HW MAP decoding performs without warm-up scheme and accessing rim state metrics scheme. 
a

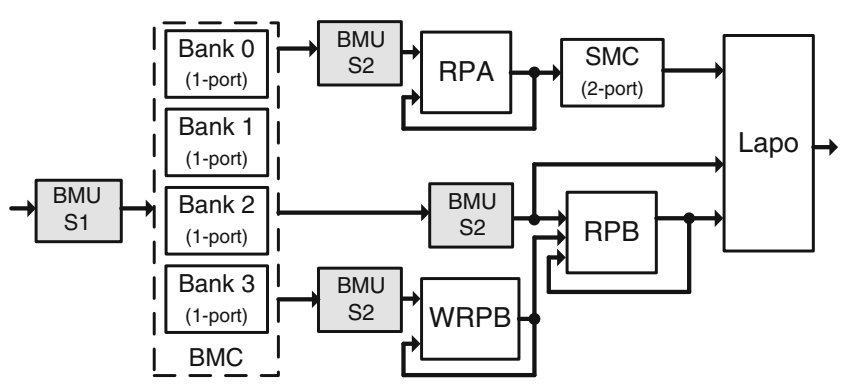

b

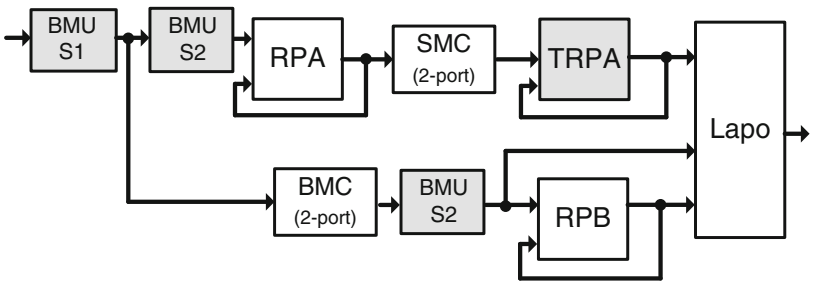

Figure 10 Block diagram of a warm-up HW MAP processor and $\mathbf{b}$ WFHW MAP processor.

solution is to use the same address decoder for all memory banks. This vectorizable property requires the interleaving address satisfying

$(\pi(t+j W))_{\bmod W}=(\pi(t))_{\bmod W}$,

where $0 \leqq t<W$, and $0 \leqq j<P$. The equality implies that each MAP processor accesses data based on the same local address. Based on this vectorizable property, only one decoder is required. All memory banks can merge into a single physical memory with data stored and fetched as vectors as shown in Fig. 5.

A high-level simulation model is constructed to analyze the available parallelism for the LTE-based and WiMAXbased CTC decoding with $24 \leqq W \leqq 36$. Then, we achieve $P$ MAP processors and $S$ number of decoding window for each MAP processor. Table 2 lists the available parallelism achieving the collision-free and vectorizable interleaving. To design the proposed dual-standard CTC decoder, the parallelism $P$ is set to 8 for the WiMAX-2 standard and 16 for the LTE-A standard. Figure 6 shows the overall architecture of the proposed collision-free vectorizable dualstandard parallel interleaver and the CTC controller. The CTC controller is used to provide control signals and initial parameters. To perform the radix-4 SB/DB MAP decoding, the proposed dual-standard address generators generate the WiMAX-based addresses or the LTE-based even addresses by adopting a hardware sharing technique. The additional LTE address generators generate the LTE-based odd addresses in the LTE modes. The LTE-based and WiMAXbased interleaving parameters $(P 0, T P 1, T P 2, T P 2, P(0)$, $H(0), J(0)$, and $\left.f_{2}\right)$ can be implemented in two parameter read-only memories (PROMs). The address decoder transforms the interleaving addresses into the collision-free addresses of the memory banks. Table 3 shows total gate counts of the proposed parallel dual-standard interleaver and CTC controller. Compared with the LTE address generators, the overhead of the proposed dual-standard address generators is about $1.2 \mathrm{~K}$ gates in order to support the WiMAX interleaving. Besides, the address decoders are less dominant in the design of the dual-standard parallel interleaver and CTC controller.

\subsection{Circular Parallel MAP Decoding}

To achieve the circular parallel MAP decoding, we first introduce the methods to achieve initial forward and backward state metrics of each frame for the distinct trellis terminations described in Section 2.1. The initial forward and backward state metrics of each frame can be attained by two distinct methods for the WiMAX-based standard and LTE-based standard. The method used in WiMAX-based standard is the circular encoding [21], which ensure the ending trellis state equals the initial trellis state. Thus, the initial values of the forward state metrics $\alpha_{0}^{k}(s)$ and backward
Table 4 Area evaluations of the Radix-4 SB/DB Warm-up HW MAP processor and WFHW MAP processor (90 nm Process@150 MHz).

\begin{tabular}{lcc}
\hline Module & Warm-up HW MAP $\left(\mu \mathrm{m}^{2}\right)$ & WFHW MAP $\left(\mu \mathrm{m}^{2}\right)$ \\
\hline BMU S1 & 1095.40 & 1095.40 \\
BMC & $49862.52(4$ single-port SRAMs $)$ & 13496.44 (1 dual-port SRAM) \\
BMU S2s & 9597.11 & 6398.07 \\
RPB & 12500.71 & 12500.71 \\
WRPB & 12500.71 & - \\
RPA & 12499.69 & 12499.69 \\
SMC & $17892.10($ dual-port SRAM $)$ & 14510.82 (dual-port SRAM) \\
TRP & - & 3379.92 \\
LAPO & 24678.65 & 24678.65 \\
RMC & - & 25013.21 (2 single-port SRAMs) \\
Overall & $140626.89(100 \%)$ & 113572.91 (80.76 \%) \\
\hline
\end{tabular}


state metrics $\beta^{k}{ }_{N}(s)$ of the $k^{\text {th }}$ iteration for the circular encoding can be determined by

$\left\{\begin{array}{c}\alpha_{0}^{k}(s)=\left\{\begin{array}{c}0(\text { for } \mathrm{k}=1), \\ \alpha_{N}^{k-1}(s)(\text { others }),\end{array}\right. \\ \beta_{N}^{k}(s)=\left\{\begin{array}{c}0(\text { for } \mathrm{k}=1), \\ \beta_{0}^{k-1}(s)(\text { others }),\end{array}\right.\end{array}\right.$

where $\alpha^{k-1}{ }_{N}(s)$ and $\beta^{k-1}{ }_{0}(s)$ are the final state metrics of the $(k-1)^{\text {th }}$ iteration. The method used in LTE-based standard is to force the trellis terminated at the state 0 . Thus, the initial values of $\alpha^{k}{ }_{0}(s)$ and $\beta_{N}^{k}(s)$ for the 0 -state trellis termination can be determined by

$\left\{\begin{array}{c}\alpha_{0}^{k}(0)=\beta_{N}^{k}(0)=0 \\ \alpha_{0}^{k}\left(s^{\prime}\right)=\beta_{N}^{k}\left(s^{\prime}\right)=\infty\end{array}\right.$

where $s^{\prime}$ denotes the states that are not state 0 .

We then introduce the WFHW MAP decoding of the circular parallel MAP decoding. Figure 7(b) shows the overall timing chart of the WFHW MAP decoding, which is composed of the basic WFHW window shown in Fig. 7(a). The key concept is to introduce the initial rim forward and backward state metrics of the $(k+1)^{\text {th }}$ iteration to the HW decoding by utilizing the final rim forward and backward state metrics of the $k^{\text {th }}$ iteration. Instead of performing a warm-up recursion, each WFHW MAP processor achieves the initial rim state

Utilization Ratio $=84.3 \%$ RAM $\left(3072 \times 2 n_{e x}\right)$

\begin{tabular}{|c|c|c|}
\hline : & $\vdots$ & $\begin{array}{c}\text { RAM } \\
\left(2400 \times n_{e x}\right)\end{array}$ \\
\hline $\operatorname{Lex}^{01}{ }_{2399}$ & $\operatorname{Lex}^{10} 2399$ & $\operatorname{Lex}^{11}{ }_{2399}$ \\
\hline : & : & : \\
\hline $\operatorname{Lex}^{01}{ }_{2}$ & $\operatorname{Lex}^{10}{ }_{2}$ & $\operatorname{Lex}^{11}{ }_{2}$ \\
\hline $\operatorname{Lex}^{01}{ }_{1}$ & $\operatorname{Lex}^{10}{ }_{1}$ & $\operatorname{Lex}^{11}{ }_{1}$ \\
\hline $\operatorname{Lex}^{01}{ }_{0}$ & $\operatorname{Lex}^{10}{ }_{0}$ & $\operatorname{Lex}^{11}{ }_{0}$ \\
\hline
\end{tabular}
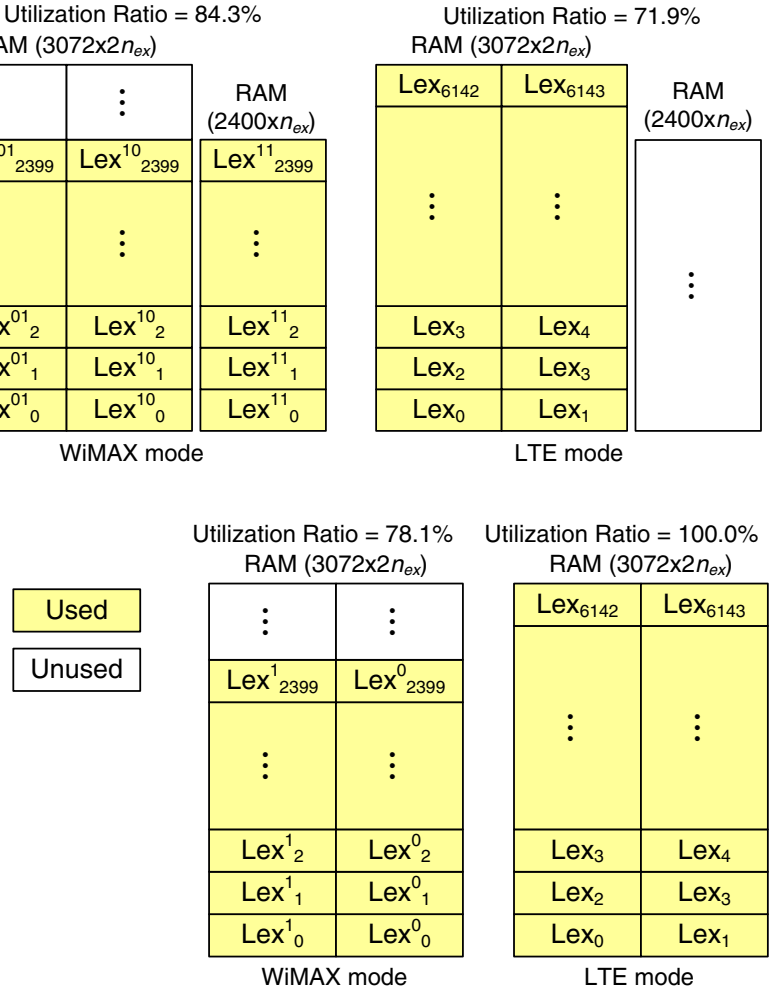

Figure 11 a Two-bank and $\mathbf{b}$ one-bank extrinsic buffer designs for the LTE/WiMAX standards.

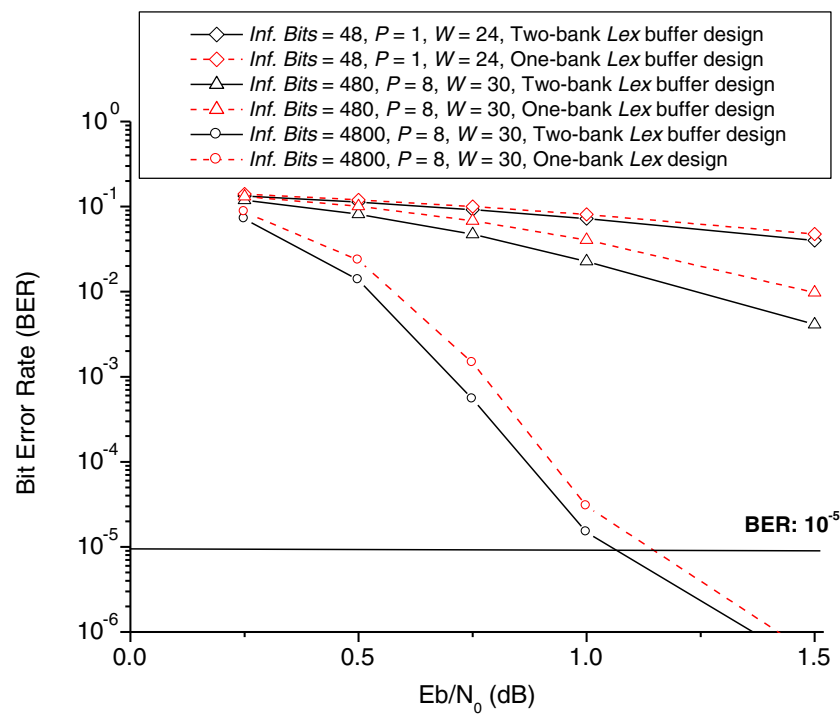

Figure 12 BER performance of the two extrinsic buffer designs for WiMAX-2 CTC schemes by using the circular parallel MAP decoding at fixed 6 iterations.

metrics of current iteration by fetching the final rim state metrics of previous iteration from the rim metrics cache (RMC). The initial forward and backward state metrics of the $(k+1)$ th iteration can be determined by

$$
\left\{\begin{array}{c}
\alpha_{j \cdot\lfloor N / P\rfloor}^{k+1}(s)=\alpha_{j \cdot\lfloor N / P\rfloor}^{k}(s), \\
\beta_{t \cdot W}^{k+1}(s)=\beta_{t \cdot W}^{k}(s),
\end{array}\right.
$$

where $1 \leqq k \leqq$ maximum number of iteration $I, 0 \leqq t<W$, and $0 \leqq j<P$. Then, Fig. 8 shows the circular trellis propagation of backward recursion of the circular parallel MAP decoding as the iteration is increased. The forward recursion can achieve the same effect in the opposite direction. Thus, the circular parallel MAP decoding results in $P$ number of circular MAP decoding as iteration increases. Instead of performing warmup recursion to get the initial state metrics, the WFHW MAP processor can fetch it from RMC so that the decoding latency can reduce to $1 \mathrm{~W}$. The throughput rates can be improved $18.2 \%$ for $N=2400$ WiMAX-2 CTC decoding and $15 \%$ for $N=3072$ LTE-A CTC decoding compared to the warm-up HW MAP decoding [12]. Figure 9 presents the floatingpoint simulation results of distinct HW MAP decoding for $N=2400$ WiMAX-2 and $N=6144$ LTE-A CTC schemes with $P=8$ at fixed 6 iterations. The circular parallel MAP decoding

Table 5 Area and power evaluations of the two-bank and one-bank extrinsic buffer designs (90 nm Process@150 MHz).

\begin{tabular}{lrr}
\hline Design & $\begin{array}{l}\text { Two-bank extrinsic } \\
\text { buffer design }\end{array}$ & $\begin{array}{l}\text { One-bank extrinsic } \\
\text { buffer design }\end{array}$ \\
\hline Area $\left(\mu \mathrm{m}^{2}\right)$ & $307449.50(100 \%)$ & $204184.26(66.41 \%)$ \\
Power $(\mathrm{mW})$ & $12.79(100 \%)$ & $8.86(69.27 \%)$ \\
\hline
\end{tabular}




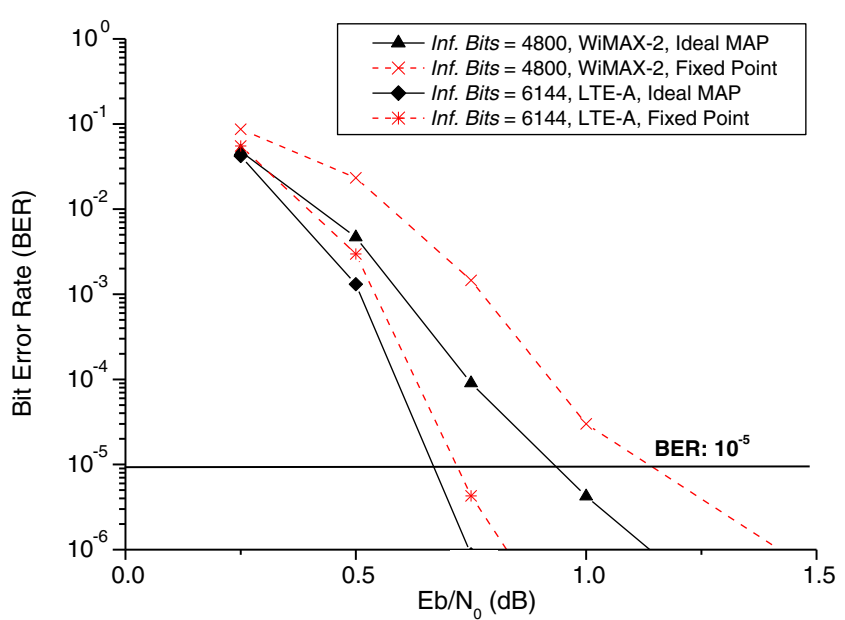

Figure 13 BER performance of the CTC decoding by using the prototyping CTC decoder chip.

greatly improves the coding gain compared with the no warmup HW MAP decoding. Due to the less reliability of extrinsic information exchange, however, the coding gain loss of the circular parallel MAP decoding is less than $0.1 \mathrm{~dB}$ at a BER of $10^{-5}$ compared with the warm-up HW MAP decoding.

Figure 10 shows the block diagram of warm-up HW MAP processor and WFHW MAP processor which are based on the timing chart shown in Figs. 3 and 7, respectively. In order to achieve a high area usage, we apply the radix-4 SB/DB EMLMAP decoding modules [12] to the both MAP processors. The six temporary terms of branch metrics calculated by the first-stage branch metrics unit (BMU S1) are stored into the branch metrics cache (BMC), and then the sixteen branch

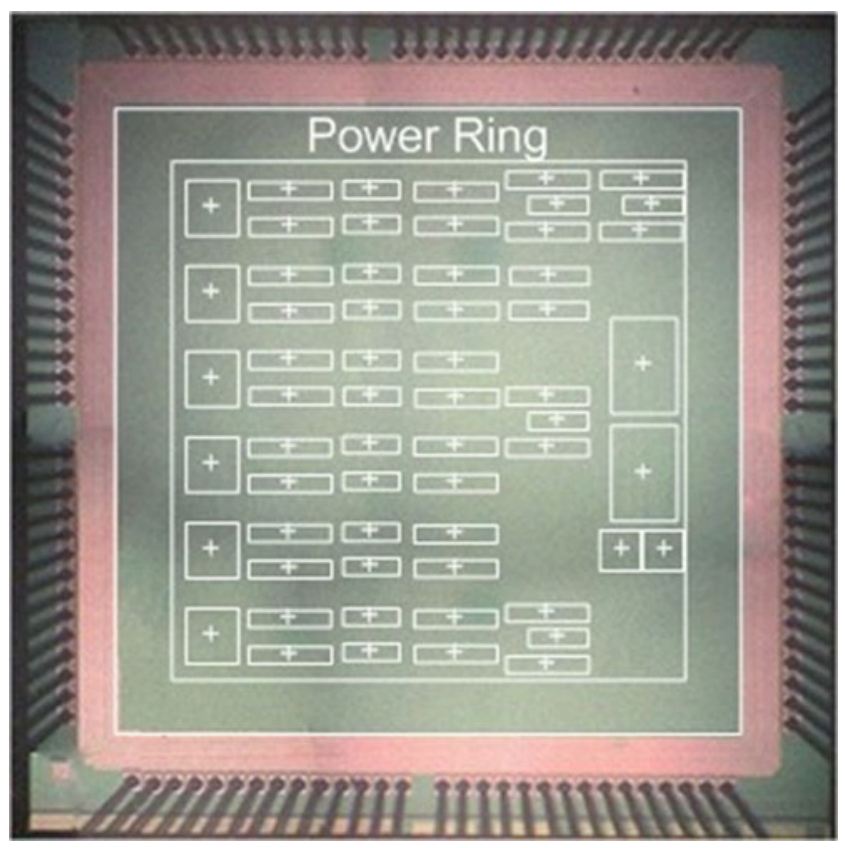

Figure 14 Die photo of the prototyping CTC decoder chip. SRAM block).
Table 6 Summary of the prototyping CTC decoder chip.

\begin{tabular}{ll}
\hline Technology & UMC 90 nm CMOS \\
\hline Max. frequency & $152 \mathrm{MHz}$ \\
Core size & $1.84 \times 1.83 \mathrm{~mm}^{2}$ \\
Max. throughput rate $\boldsymbol{T} @ N=6144,6$ iterations & $186.1 \mathrm{Mbps}$ \\
Power consumption@ $\boldsymbol{T}$ & $148.1 \mathrm{~mW}$ \\
\hline
\end{tabular}

metrics are fetched by the second-stage branch metrics unit (BMU S2). Based on the basic window shown in Fig. 7(a), one set of BMU S1 and two sets of BMU S2 are required for the WFHW MAP decoding. The forward recursion processing element (RPA), warm-up backward recursion processing element (WRPB), and backward recursion processing element (RPB) are composed of radix-4 add-compare-select units (ACSUs). The forward traceback recursion processing element (TRPA) composed of radix-4 traceback units [23] are adopted to reduce the access power of the state metrics cache (SMC). Finally, a posteriori LLR module (Lapo) is used to compute a-posteriori LLR. Table 4 lists the area evaluation obtained by using the $90 \mathrm{~nm}$ CMOS process based on the quantization scheme referred to in Table 3 in [12] with $W=36$ and $S=16$. The RMC is evaluated with the WFHW MAP processor to correctly perform the CTC decoding. The RMC is composed of two single-port SRAMs because each SRAM stores the rim state metrics of half iteration. Even the hardware cost of RMC is large. The WFHW MAP processor achieves

Table 7 Parameters and throughputs of the prototyping CTC decoder.

\begin{tabular}{|c|c|c|c|c|c|}
\hline \multicolumn{2}{|l|}{ Info. Bits } & \multirow[t]{2}{*}{ Active MAPs } & \multirow[t]{2}{*}{$S$} & \multirow[t]{2}{*}{$W$} & \multirow{2}{*}{$\begin{array}{l}\text { Throughput } \\
\text { (Mbps@152 MHz) }\end{array}$} \\
\hline WiMAX-2 & LTE-A & & & & \\
\hline 48 & 48 & 1 & 1 & 24 & 10.8 \\
\hline 72 & 72 & 1 & 1 & 36 & 11.4 \\
\hline 96 & 96 & 2 & 1 & 24 & 21.7 \\
\hline 144 & 144 & 2 & 1 & 36 & 32.5 \\
\hline 192 & 192 & 4 & 1 & 24 & 43.4 \\
\hline 216 & 216 & 3 & 1 & 36 & 34.2 \\
\hline 240 & 240 & 4 & 1 & 30 & 44.7 \\
\hline 288 & 288 & 4 & 1 & 36 & 65.2 \\
\hline 360 & 360 & 6 & 1 & 30 & 67.0 \\
\hline 384 & 384 & 8 & 1 & 24 & 86.8 \\
\hline 432 & 432 & 8 & 1 & 27 & 88.3 \\
\hline 480 & 480 & 8 & 1 & 30 & 89.5 \\
\hline 960 & 960 & 8 & 2 & 30 & 124.0 \\
\hline 1920 & 1920 & 8 & 4 & 30 & 153.9 \\
\hline 2880 & 2880 & 8 & 6 & 30 & 167.3 \\
\hline 3840 & 3840 & 8 & 8 & 30 & 175.0 \\
\hline 4800 & 4800 & 8 & 10 & 30 & 179.3 \\
\hline- & 6144 & 8 & 16 & 24 & 186.1 \\
\hline
\end{tabular}


area reduction because the hardware cost of WRPB and BMC are majorly reduced compared with the warm-up HW MAP processor. Thus, the proposed CTC decoder for WiMAX-2 and LTE-A standards adopts the circular parallel MAP decoding with the WFHW MAP processor shown in Fig. 10(b).

\subsection{Efficient WiMAX/LTE Extrinsic Buffer Design}

Because of the radix-4 DB MAP decoding, three extrinsic information values are accessed in an extrinsic buffer in one cycle. The conventional radix-4 DB CTC decoder requires $2400 \times 3 n_{e x}$ SRAM to store the three extrinsic information values for WiMAX standard, where $n_{e x}$ denotes the bitlength of an extrinsic information value. Meanwhile, two extrinsic information values are accessed in an extrinsic buffer in one cycle because of the radix-4 SB MAP decoding. Thus, the conventional radix-4 SB CTC decoder requires $3072 \times 2 n_{e x}$ SRAM to store the extrinsic information values for LTE standard. In order to increase the hardware usage of extrinsic buffer, Fig. 11(a) shows an efficient two-bank extrinsic buffer design for the dual standards. Compared with a one-bank

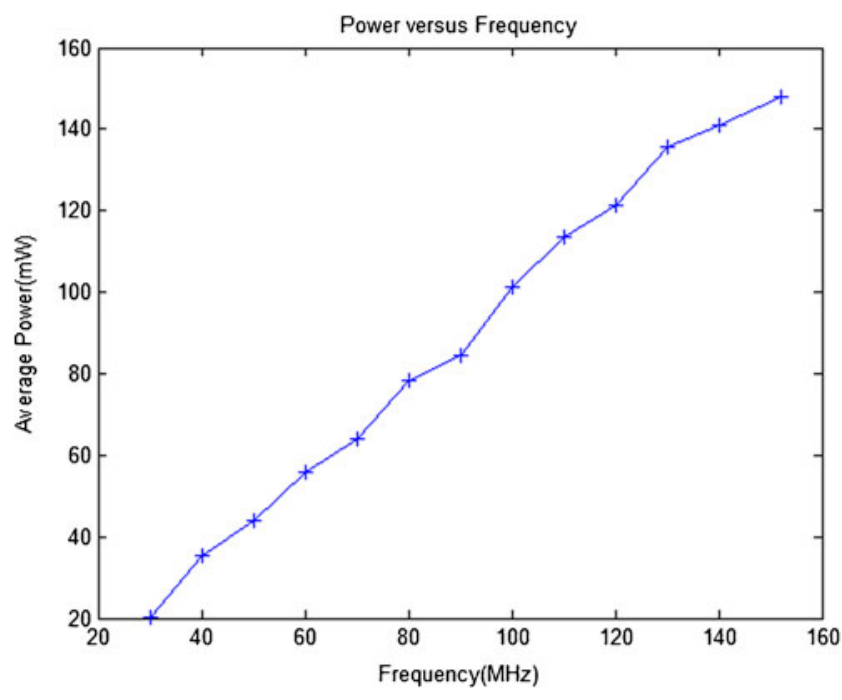

Figure 16 Measured power with different operating frequencies at core $\mathrm{vdd}=1.1 \mathrm{~V}$.

$3072 \times 3 n_{e x}$ SRAM design, this two-bank extrinsic buffer design can reduce the size of extrinsic buffer. The two-bank

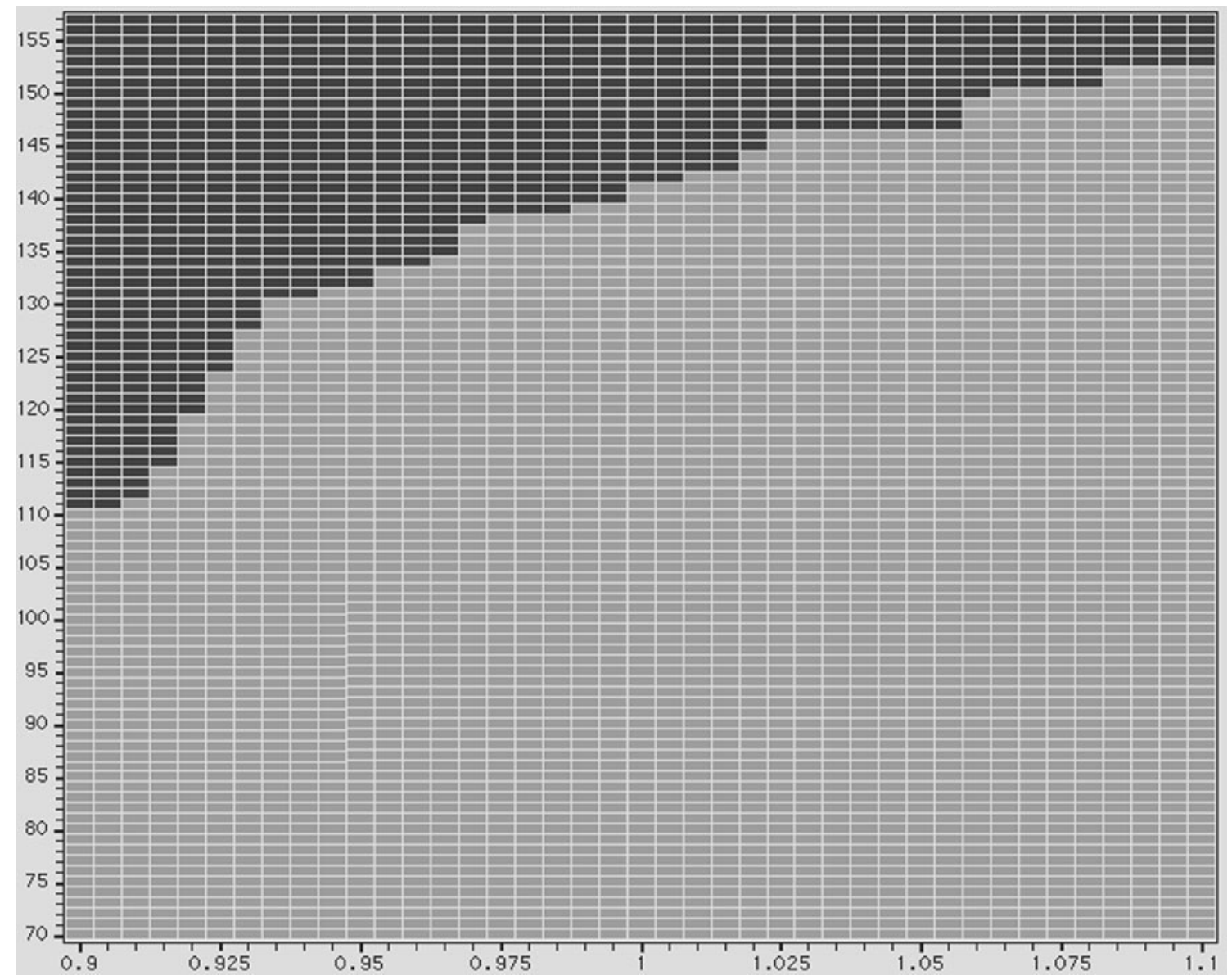

Figure 15 Shmoo plot of chip testing. This plot is captured by an Agilent 93000 SOC Series Test System. X-axis denotes core vdd from $0.9 \mathrm{~V}$ to $1.1 \mathrm{~V}$, and Y-axis denotes frequency from $70 \mathrm{MHz}$ to $155 \mathrm{MHz}$. Light-gray block means that the chip passes the testing, and dark-gray block means that the chip does not pass the testing. 


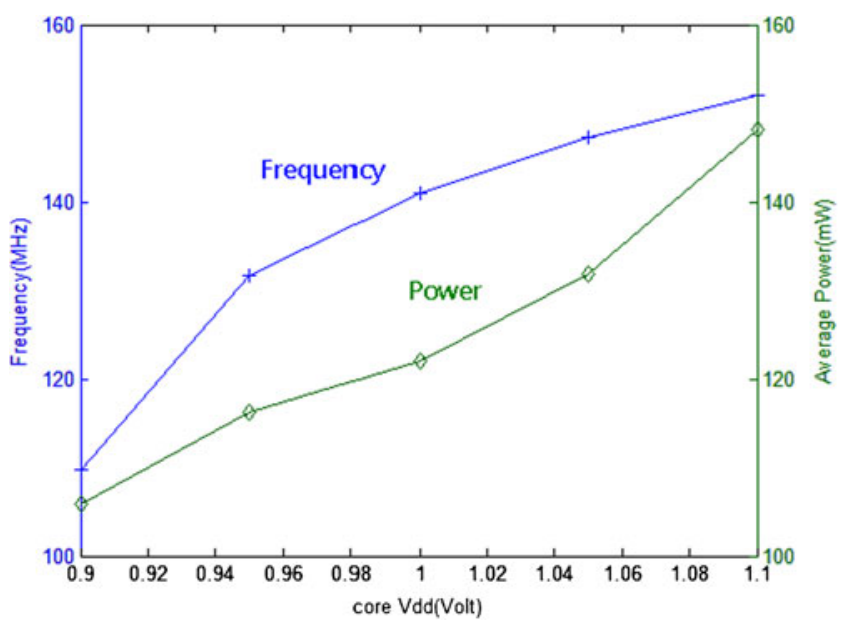

Figure 17 Measured frequency and power of the prototyping CTC decoder chip.

extrinsic buffer design can disable the $2400 \times n_{e x}$ SRAM in LTE mode to reduce the power consumption. To further increase the hardware usage of extrinsic buffer, Fig. 11(b) shows an efficient extrinsic buffer design by using the bit-level extrinsic information exchange method [22]. This method transfers the extrinsic information of radix-4 DB MAP decoding from symbol-level values to bit-level values and makes the extrinsic buffer access only two extrinsic information values. Thus, the extrinsic buffer for the dual standards can be implemented by a one-bank $3072 \times 2 n_{e x}$ SRAM and achieve $100 \%$ utilization ratio in LTE mode. Figure 12 illustrates the floatingpoint simulation results of the two extrinsic buffer designs for WiMAX-2 CTC schemes by using the circular parallel MAP decoding at fixed 6 iterations. The two-bank extrinsic buffer design loses less than $0.2 \mathrm{~dB}$ coding gain at a BER of $10^{-5}$ due to the bit-level extrinsic information exchange method for the WiMAX-2 CTC decoding. Compared to the aforementioned two-bank design, the one-bank design with the bit-level extrinsic information exchange method reduces $28.1 \%$ size of the extrinsic buffer and eliminates the $2400 \times n_{e x}$ SRAM. Table 5 lists the area evaluation obtained by using the $90 \mathrm{~nm}$ CMOS process with $n_{e x}=9$. Compared with the two-bank extrinsic buffer design, the one-bank extrinsic buffer design achieves a low area cost and low power consumption. Hence, the proposed CTC decoder for WiMAX-2 and LTE-A standards adopts the efficient extrinsic buffer design with the bitlevel extrinsic information exchange method.

\section{CHIP Implementation and Comparisons}

The design of the CTC decoder is simulated using C-to-RTL flow with the quantization scheme referred to in Table 3 in [12]. The parameters of the proposed CTC decoder can meet the targeted BER of $10^{-5}$ for WiMAX-2 and LTE-A standards. Figure 13 shows the simulated BER performance of the distinct CTC schemes decoded by the prototyping CTC decoder based on additive white Gaussian noise (AWGN) channels and 6 iterations. The ideal MAP represents the floating-point CTC decoding with knowing the initiate trellis states and without the windowing technique. The fixpoint represents the fixed-point CTC decoding by using the proposed CTC decoder for the LTE-A and WiMAX-2 standards.

\subsection{Prototyping Chip Implementation and Measurement Results}

The proposed CTC decoder has been implemented in an ASIC by using Verilog HDL codes synthesized with the standard cell library of UMC $90 \mathrm{~nm} \mathrm{1P9M} \mathrm{CMOS} \mathrm{process}$

Table 8 Comparisons of the CTC decoder chips.

\begin{tabular}{|c|c|c|c|c|c|}
\hline Publication & {$[24]$} & {$[26]$} & {$[25]$} & {$[27]$} & Proposed \\
\hline Technology & $130 \mathrm{~nm}$ & $130 \mathrm{~nm}$ & $130 \mathrm{~nm}$ & $90 \mathrm{~nm}$ & $90 \mathrm{~nm}$ \\
\hline Core voltage & $1.2 \mathrm{~V}$ & $1.2 \mathrm{~V}$ & $1.2 \mathrm{~V}$ & $1 \mathrm{~V}$ & $1.1 \mathrm{~V}$ \\
\hline Standard & UMTS, HSDPA & Mobile WiMAX & LTE-A, WiMAX & LTE & LTE-A, WiMAX-2 \\
\hline Max. Info. Bits & 5144 & 4800 & 6144 & 6144 & 6144 \\
\hline MAP & Radix-2 SB & Radix-4 DB & Radix-4 SB/DB & Radix-2 SB & Radix-4 SB/DB \\
\hline Parallelism $\boldsymbol{P}$ & 1 & 1 & 8 & 8 & 8 \\
\hline Max. frequency (MHz) & 246 & 200 & 250 & 275 & 152 \\
\hline Core area $\left(\mathrm{mm}^{2}\right)$ & 1.20 & 2.24 & 10.7 & 2.10 & 3.38 \\
\hline Max. throughput $\boldsymbol{T}$ (Mbps) & $20.2 @ 5.5$ iter. & $48.5 @ 8$ iter. & $187.5 @ 8$ iter. & $130 @ 8$ iter. & 186.1@6iter. \\
\hline Power (mW)@T & 61.5 & N/A & N/A & 219 & 148.1 \\
\hline Energy efficiency (nJ/bit/iter.) & $0.54\left(0.18^{\mathrm{a}}\right)$ & N/A & $0.61\left(0.20^{\mathrm{a}}\right)$ & 0.21 & 0.13 \\
\hline Area efficiency $\left(\mathrm{bit} / \mathrm{mm}^{2}\right)$ & $0.07\left(0.15^{\mathrm{b}}\right)$ & $0.11\left(0.23^{\mathrm{b}}\right)$ & $0.07\left(0.15^{\mathrm{b}}\right)$ & 0.23 & 0.36 \\
\hline
\end{tabular}

${ }^{\mathrm{a}}$ Normalized energy factor $=0.33\left(=(1.0 \mathrm{~V} / 1.2 \mathrm{~V})^{2} \times(90 \mathrm{~nm} / 130 \mathrm{~nm})^{2}\right)$

${ }^{\mathrm{b}}$ Normalized area factor $=2.09\left(=(130 \mathrm{~nm} / 90 \mathrm{~nm})^{2}\right)$ 
and packed in a CQFP128 package. This prototyping decoder supports the WiMAX-2 and LTE-A CTC schemes. The chip implementation of the proposed CTC decoder is obtained in a core size of $3.38 \mathrm{~mm}^{2}$ and contains $232.8 \mathrm{~Kb}$ RAM. Figure 14 shows the die photo of the proposed CTC decoder and Table 6 summarizes this chip. The chip is measured by using an Agilent 93000 system-on-a-chip (SoC) Series Test System. The chip is maximally measured at $152 \mathrm{MHz}$ operating frequency. The number of active MAP processors with the distinct design modes is shown in Table 7. At 6 iterations, the chip achieves maximum throughput rate of $179.3 \mathrm{Mbps}$ and $186.1 \mathrm{Mbps}$ for WiMAX-2 and LTE-A, respectively. Besides, Fig. 15 shows the shmoo plot with core vdd from $0.9 \mathrm{~V}$ to $1.1 \mathrm{~V}$ and operation frequency from $70 \mathrm{MHz}$ to $155 \mathrm{MHz}$. Then, Fig. 16 shows the measured power consumptions with different operation frequencies at core vdd of $1.1 \mathrm{~V}$. This plot indicates that the power consumptions are $148.1 \mathrm{~mW}$ at operation frequency of $152 \mathrm{MHz}$ and core vdd of $1.1 \mathrm{~V}$. Furthermore, to consider reduction of power consumption, the core supply voltage can be reduced from $1.1 \mathrm{~V}$ to $0.9 \mathrm{~V}$. The measured maximal operating frequencies and power consumptions are shown in Fig. 17.

\subsection{Comparisons}

In Table 8, the proposed CTC decoder for LTE-A and WiMAX-2 standards is compared with other chip designs. The works in [24] and [26] perform the radix-2 SB MAP decoding and radix-4 DB MAP decoding for HSDPA and WiMAX systems, respectively. Since the throughput rate requirements are less than $20 \mathrm{Mbps}$, both of these employ only one MAP processing. To achieve throughput rates higher than $100 \mathrm{Mbps}$, the work in [27] employs 8 MAP processors and supports LTE CTC schemes. Our proposed CTC decoder employs 8 radix-4 SB/DB WFHW MAP processors with the proposed collision-free parallel interleaver for the dual-standard CTC schemes. It is hard to compare these chips since the coding parameters are different from each other. However, we use normalized energy efficiency (NEE),

$\mathrm{NEE}=\frac{\text { Power }}{\text { Throughput } \times \text { Iteraion }} \times$ Normalized energy factor,

and normalized area efficiency (NAE),

$\mathrm{NAE}=\frac{\text { Throughput }}{\text { Area } \times \text { Frequency }} \times$ Normalized area factor

as the performance indices. The NEE indicates how much energy a decoder chip consumes to process a hard bit at an iteration. The NAE indicates how many hard bits per one $\mathrm{mm}^{2}$ for a single CTC block a decoder chip decodes. To support the high-mobility $4 \mathrm{G}$ application of the LTE-A/WiMAX-2 CTC decoding, this chip achieves a high NAE of $0.36 \mathrm{bit} / \mathrm{mm}^{2}$ with a low NEE of $0.13 \mathrm{~nJ} / \mathrm{bit} /$ iteration.

\section{Conclusion}

In this paper, a turbo decoder chip supporting distinct block sizes of convolutional turbo code schemes for the highmobility $4 \mathrm{G}$ applications of both LTE-A and WiMAX-2 systems is proposed. The circular parallel MAP decoding is introduced to achieve high throughput rate and low hardware cost. The collision-free vectorizable dual-standard parallel interleaver is proposed to enhance the hardware usage. The two efficient extrinsic buffer designs are also described in this paper to increase the memory utilization. The CTC decoder chip for LTE-A/WiMAX-2 standards is fabricated to verify the proposed techniques. This decoder chip achieves the both LTE-A and WiMAX-2 data-rate requirements with a high area efficiency and a low energy efficiency.

Acknowledgment The authors would like to thank Chip Implementation Center (CIC) for the support of chip fabrication.

\section{References}

1. Berrou, C., Glavieux, A., \& Thitimajshima, P. (1993). Near Shannon limit error-correcting coding and decoding: Turbo Codes. In Proceedings of IEEE International Conference Communications (ICC) (pp. 1064-1070).

2. 3rd Generation Partnership Project (3GPP). [Online]. Available: http://www.3gpp.org/

3. Berrou, C., \& Jezequel, M. (1999). Non-binary convolutional codes for turbo coding. Electronics Letters, 35(1), 39-40.

4. Worldwide Interoperability for Microwave Access (WiMAX). [Online]. Available: http://www.wimaxforum.org/home/

5. International Telecommunication Union. [Online]. Available: http://www.itu.int/

6. Lin, C.-H., Chen, C.-Y., \& Wu, A.-Y. (2008).High-throughput $12-$ Mode CTC decoder for WiMAX standard. In Proceedings of IEEE International Symposium on VLSI Design, Automation, and Test (VLSI-DAT) (pp. 216-219).

7. Wong, C.-C., Lee, Y.-Y., \& Chang, H.-C. (2009). A 188-size $2.1 \mathrm{~mm}^{2}$ reconfigurable turbo decoder chip with parallel architecture for 3GPP LTE system. In Proceedings of IEEE International Symposium on VLSI Circuits (VLSIC) (pp. 288-289).

8. Bahl, L. R., et al. (1974). Optimal decoding of linear codes for minimizing symbol error rate. IEEE Transactions on Information Theory, 20(2), 284-287.

9. Robertson, P., Villebrun, E., \& Hoeher, P. (1995). A comparison of optimal and sub-optimal MAP decoding algorithms operating in the log domain. In Proceedings of IEEE International Conference on Communication (ICC) (pp. 1009-1013). 
10. Papaharalabos, S., Sweeney, P., \& Evans, B. G. (2005). SISO algorithms based on combined $\max / \mathrm{max}^{*}$ operations for turbo decoding. Electronics Letters, 41(3), 142-143.

11. Vogt, J., \& Finger, A. (2000). Improving the max-log-MAP turbo decoder. Electronics Letters, 36(23), 1937-1939.

12. Lin, C.-H., Chen, C.-Y., \& Wu, A.-Y. (2011). Area-efficient scalable MAP processor design for high-throughput multistandard convolutional turbo decoding. IEEE Transactions Very Large Scale Integration (VLSI) Systems, 19(no. 2), 305-318.

13. Viterbi, A. J. (1998). An intuitive justification and simplified implementation of the MAP decoder for convolutional codes. IEEE Journal on Selected Areas in Communications, 16, 260264.

14. Masera, G., et al. (1999). VLSI architecture for turbo codes. IEEE Journal on Selected Areas in Communications, 7(no. 3), 369-379.

15. Boutillon, E., Gross, W. J., \& Gulak, P. G. (2003). VLSI architectures for the MAP algorithm. IEEE Transactions on Communications, 51(2), 175-185.

16. Kaza, J., \& Chakrabarti, C. (2004). Design and implementation of low-energy turbo decoders. IEEE Transactions on Very Large Scale Integration (VLSI) Systems, 12(no. 9), 968-977.

17. Wu, C.-M., et al. (2005). VLSI architectural design tradeoffs for sliding-window log-MAP decoders. IEEE Transactions on Very Large Scale Integration (VLSI) Systems, 13(no. 4), 439447.

18. Li, F.-M., Lin, C.-H., \& Wu, A.-Y. (2008). Unified convolutional/ turbo decoder design using tile-based timing analysis of VA/MAP kernel. IEEE Transactions on Very Large Scale Integration (VLSI) Systems, 16(no. 10), 1358-1371.

19. Lin, C.-H., Chang, E.-J., Chen, C.-Y., \& Wu, A.-Y. (2011). A 0.16 $\mathrm{nJ} /$ bit/iteration $3.38 \mathrm{~mm}^{2}$ Turbo Decoder Chip for WiMAX/LTE Standards. In Proceedings of the IEEE International Symposium on Integrated Circuits (ISIC) (pp. 178-181).

20. Nimbalke, A., et al. (2008). ARP and QPP interleavers for LTE turbo coding. In Proceedings of IEEE Wireless Communications and Networking Conference (WCNC) (pp. 1032-1037).

21. Zhan, C., et al. (2006). An efficient decoder scheme for double binary circular turbo codes. In Proceedings of IEEE International Conference on Acoustics, Speech and Signal Processing (ICASSP) (pp. 229-232).

22. Kim, J.-H., \& Park, I.-C. (2009). Bit-level extrinsic information exchange method for double-binary turbo codes. IEEE Transactions on Circuits and Systems Part II, Express Briefs., 56(1), 81-85.

23. Lin, C.-H., et al. (2009). Low-power memory-reduced traceback MAP decoding for double-binary convolutional turbo decoder. IEEE Transactions on Circuits and Systems Part I, Regular Papers, 56(5), 1005-1016.

24. Benkser, C., et al. (2009). Design and optimization of an HSDPA turbo ASIC. IEEE Journal of Solid-State Circuits, 44(1), 98-106.

25. Lim, J.-H., \& Park, I. C. (2009). A unified parallel radix-4 turbo decoder for mobile WiMAX and 3GPP-LTE. In Proceedings of IEEE Custom Integrated Circuits Conference (CICC) (pp. 487-490).

26. Kim, J.-H., \& Park, I.-C. (2008). A 50Mbps double-binary turbo decoder for WiMAX based on bit-level extrinsic information exchange. In Proceedings of IEEE Asian Solid-State Circuits Conference (A-SSCC) (pp. 305-308).

27. Wong, C.-C., \& Chang, H.-C. (July 2010). Reconfigurable turbo decoder with parallel architecture for 3GPP LTE System.
Transactions on Circuits and Systems II: Express Briefs, vol. 57, no. 7.

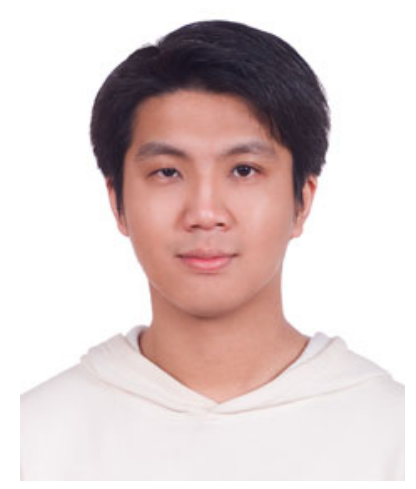

Cheng-Hung Lin received the B.S. degree in electronic engineering from Fu Jen Catholic University, Taipei, Taiwan, in 2002, M.S. degree in electrical engineering from National Central University, Taoyuan, Taiwan, in 2004, and Ph.D. degree from National Taiwan University, Taipei, Taiwan, in 2009.

In August 2000, he joined the faculty of the Department of Electrical Engineering, Yuan Ze University, Jungli, Taiwan, where he is currently an Assistant Professor. His research interests include the design of very large-scale integration architectures and circuits for digital signal processing and communication systems.

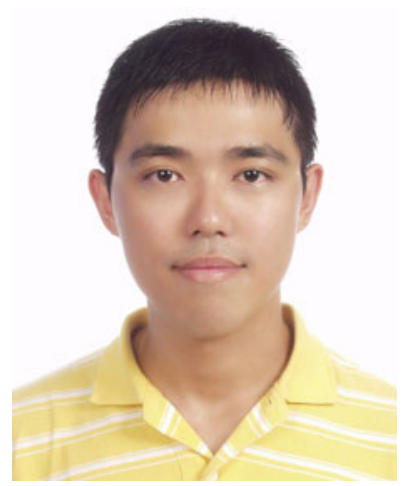

Chun-Yu Chen received the B.S. degree in electronic engineering from National Chiao Tung University, Hsinchu, Taiwan, in 2007, and M.S. degree in electronic engineering from National Taiwan University, Taipei, Taiwan, in 2009.

$\mathrm{He}$ is currently an engineer with Silicon Motion Technology Corp., Taipei, Taiwan. His research interests include the design of very largescale integration architectures and circuits for digital signal processing and communication systems. 


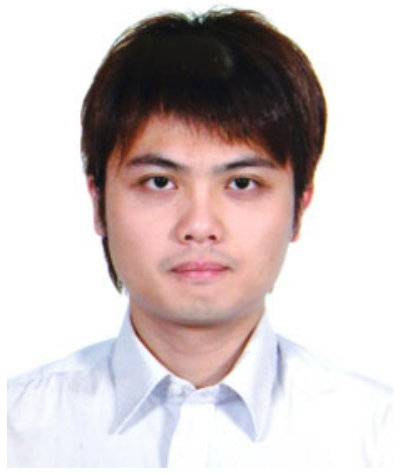

En-Jui Chang received the B.S. degree in electrical engineering from National Central University, Jungli, Taiwan, in 2008. He is currently pursuing the Ph.D. degree in the Graduate Institute of Electronics Engineering, National Taiwan University, Taipei, Taiwan.

His research interests include the design of Network-on-Chip (NoC) algorithms/architectures and very large-scale integration architectures/ circuits for digital signal processing in communication systems. He is currently working on the hardware design for coding systems.

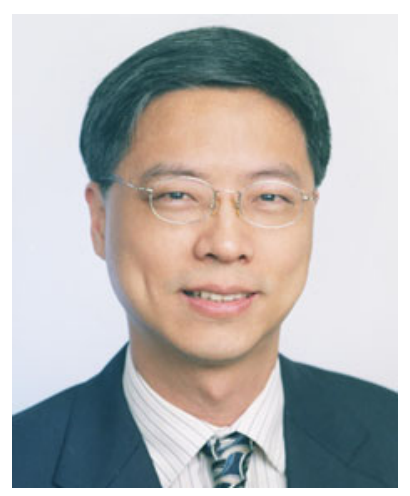

An-Yeu (Andy) Wu received the B.S. degree from National Taiwan University in 1987, and the M.S. and Ph.D. degrees from the University of Maryland, College Park in 1992 and
1995, respectively, all in Electrical Engineering. In August 2000, he joined the faculty of the Department of Electrical Engineering and the Graduate Institute of Electronics Engineering, National Taiwan University (NTU), where he is currently a Professor. His research interests include low-power/high-performance VLSI architectures for DSP and communication applications, adaptive/multirate signal processing, reconfigurable broadband access systems and architectures, and System-onChip (SoC)/Network-on-Chip (NoC) platform for software/hardware co-design.

Dr. Wu had served as the Associate Editors of IEEE Transactions in the circuits and systems area and signal processing area, such as IEEE TRANSACTIONS ON VERT LARGE SCALE INTEGRATION (VLSI) SYSTEMS, IEEE TRANSACTIONS ON CIRCUITS AND SYSTEMS I: REGULAR PAPERS, IEEE TRANSACTIONS ON CIRCUITS AND SYSTEMS II: EXPRESS BRIEFS, and IEEE TRANSACTIONS ON SIGNAL PROCESSING. Dr. Wu is now serving as an Associate Editor for JOURNAL of SIGNAL PROCESSING SYSTEMS (JSPS), and acted as the Lead Guest Editor of the Special Issue of "2010 IEEE Workshop on Signal Processing Systems (SiPS) in JSPS, which was published in Nov. 2011. He also served on the technical program committees of many major IEEE International Conferences, such as SiPS, AP-ASIC, ISCAS, ISPACS, ICME, SOCC, and A-SSCC. He is now serving as the Chair of VLSI Systems and Architectures (VSA) Technical Committee in IEEE Circuits and Systems (CAS) Society.

From August 2007 to Dec. 2009, he was on leave from NTU and served as the Deputy General Director of SoC Technology Center (STC), Industrial Technology Research Institute (ITRI), Hsinchu, TAIWAN, supervising Parallel Core Architecture (PAC) VLIW DSP Processor and Multicore/Android SoC platform projects. In 2010, Dr. Wu received "Outstanding EE Professor Award" from The Chinese Institute of Electrical Engineering (CIEE), Taiwan. Starting from Aug. 2012, Dr. Wu is serving as the Deputy Director of Graduate Institute of Electronics Engineering (GIEE) of National Taiwan University. 